\title{
Utilização da safena magna in situ para arterialização do arco venoso do pé
}

\section{The great saphenous vein in situ for the arterialization of the venous arch of the foot}

Cesar Roberto Busato', Carlos Alberto Lima Utrabo², Ricardo Zanetti Gomes ${ }^{3}$, Eliziane Hoeldtke², Joel Kengi Housome², Dieyson Martins de Melo Costa², Cintia Doná Busato ${ }^{4}$

\section{Resumo}

Contexto: $O$ tratamento da isquemia crítica de membros inferiores sem leito arterial distal pode ser realizado por meio da inversão do fluxo no arco venoso do pé.

Objetivo: O objetivo deste trabalho foi apresentar a técnica e os resultados obtidos com a arterialização do arco venoso do pé, mantendo a safena magna in situ.

Métodos: Dezoito pacientes, dos quais 11 com aterosclerose (AO), 6 com tromboangeíte obliterante (TO) e 1 com trombose de aneurisma de artéria poplítea (TA) foram submetidos ao método. A safena magna in situ foi anastomosada à melhor artéria doadora. $\mathrm{O}$ fluxo arterial derivado para o sistema venoso progride por meio da veia cujas válvulas são destruídas. As colaterais da veia safena magna são ligadas desde a anastomose até o maléolo medial, a partir do qual são preservadas.

Resultados: Dos pacientes, 10 (55,6\%) mantiveram suas extremidades, 5 com AO e 5 com TO; 7 (38,9\%) foram amputados, 5 com AO, 1 com TO e 1 com Ta; houve 1 óbito (5,5\%).

Conclusão: A inversão do fluxo arterial no sistema venoso do pé deve ser considerada para salvamento de extremidade com isquemia crítica sem leito arterial distal.

Palavras-chave: Tromboangeíte obliterante; salvamento de membro; arterialização temporal; amputação de membro.

\begin{abstract}
Background: Critical lower limb ischemia in the absence of a distal arterial bed can be treated by arterialization of the venous arch of the foot. Objetive: The objective of this paper was to present the technique and the results of the arterialization of the venous arch of the foot with the in situ great saphenous vein.

Methods: Eighteen patients, 11 with atherosclerosis (AO), 6 with thromboangiitis obliterans (TO) and 1 with popliteal artery aneurysm thrombosis were submitted to venous arch arterialization. The in situ great saphenous vein was anastomosed to the best donor artery. Arterial flow derived from the venous system progresses through the vein whose valves were destroyed. The collateral vessels of the great saphenous vein are linked from the anastomosis to the medial malleolus and preserved from this point onward.

Results: Limb salvage was achieved in 10 (55.6\%) patients, 5 with AO and 5 with TO. Seven (38.9\%) patients were amputated, 5 with AO, 1 with TO and 1 with Ta. One (5.5\%) patient died.

Conclusion: Arterialization of the venous system of the foot should be considered for the salvage of limbs with critical ischemia in the absence of a distal arterial bed.
\end{abstract}

Keywords: Thromboangiitis obliterans; limb salvation; temporal arterialization; limb amputation.

Trabalho realizado no Serviço de Cirurgia Vascular da Santa Casa de Misericórdia de Ponta Grossa , Ponta Grossa (PR), Brasil.

1. Professor Associado da Universidade Estadual de Ponta Grossa (UEPG); Chefe do Serviço de Cirurgia Vascular da Santa Casa de Misericórdia de Ponta Grossa, Ponta Grossa (PR), Brasil.

2. Cirurgião Vascular da Santa Casa de Misericórdia de Ponta Grossa, Ponta Grossa (PR), Brasil.

3. Professor Adjunto da UEPG; Cirurgião Vascular da Santa Casa de Misericórdia de Ponta Grossa, Ponta Grossa (PR), Brasil.

4. Acadêmico Estagiário da Faculdade Evangélica do Paraná (Fepar), Curitiba (PR), Brasil.

Não foram declarados conflitos de interesse associados à publicação deste artigo

Apresentado no VII Encontro São Paulo de Cirurgia Vascular e Endovascular, São Paulo - São Paulo

Apresentado no XXI Conclave Científico de Acadêmicos de Medicina, Cuririba - Paraná

Submetido em: 24.2.2010. Aceito em: 31.5.2010

J Vasc Bras. 2010;9(3):119-123. 


\section{Introdução}

Em isquemia crítica, sem leito arterial distal, um dos modos de irrigar o membro isquêmico é derivar o fluxo de maneira retrógrada por meio do sistema venoso, com a finalidade de tratar dor em repouso ou promover a cicatrização de úlceras e amputações.

A aterosclerose obliterante ( $\mathrm{AO})$, especialmente aquela acompanhada de diabetes melittus, a tromboangeíte obliterante (TO), na maioria absoluta dos seus casos, e o aneurisma de artéria poplítea, com trombose do leito distal, são condições que encontram indicação para o procedimento.

As primeiras tentativas de fístulas arteriovenosas terapêuticas datam do início do século passado. Realizadas na parte proximal dos membros inferiores, não obtiveram resultados favoráveis. A partir da década de 1970, com os trabalhos pioneiros de Lengua ${ }^{1}$, as fístulas passaram a ser estendidas até o pé, e os bons resultados apareceram em várias publicações, utilizando-se a veia safena magna rever$\mathrm{sa}^{2-16} \mathrm{e}$ in situ $^{11,17-20}$.

\section{Objetivo}

O objetivo deste trabalho foi apresentar a técnica e os resultados obtidos com a arterialização do arco venoso do pé, mantendo a safena magna in situ.

\section{Métodos}

A arteriografia e o mapeamento dúplex arterial foram realizados como exames de rotina à procura de leito para tratamento por enxerto convencional e para escolha da melhor artéria doadora. $\mathrm{O}$ mapeamento dúplex venoso estudou e marcou preferencialmente a safena magna e sua extensão até o arco venoso do pé, bem como a permeabilidade das demais veias do sistema venoso profundo que garantiram o retorno do hiperfluxo gerado pela confecção da fístula.

A safena magna foi anastomosada de maneira términolateral à melhor artéria doadora (Figura 1).

O fluxo arterial derivado para o sistema venoso progrediu através da veia, cujas válvulas foram destruídas pelo valvulótomo de Mills (Otemac $\mathrm{R}$ ) introduzido através de colaterais, até a altura do maléolo medial (Figura 2).

Nesse ponto, encontrou-se invariavelmente a veia perfurante anterior do maléolo, a partir da qual todas as colaterais do pé foram preservadas. Por meio de flebotomia no arco venoso dorsal, ao nível do primeiro espaço interdigital, completou-se a destruição das válvulas, o que permitiu o afluxo de sangue arterial ao dorso do pé (Figura 3).

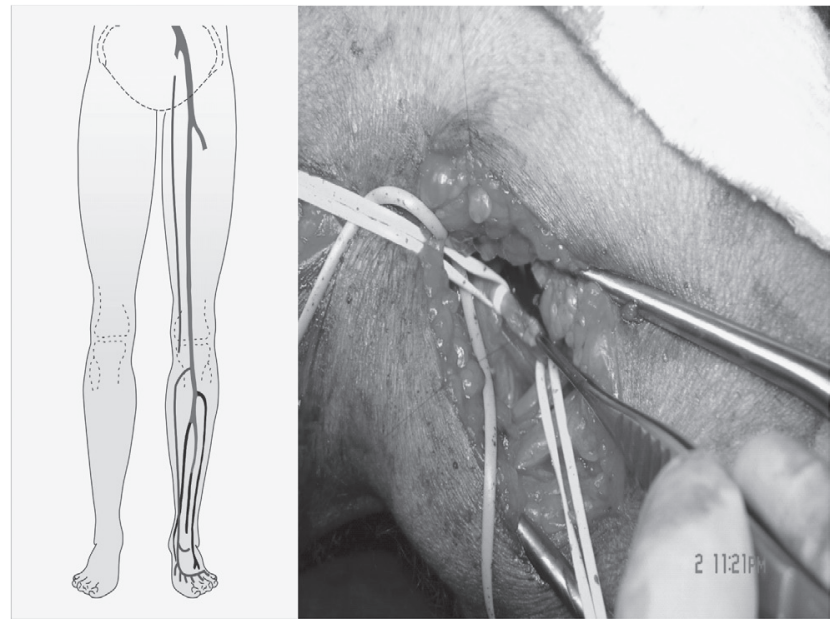

Figura 1 - Veia safena magna em anastomose término-lateral à melhor artéria doadora

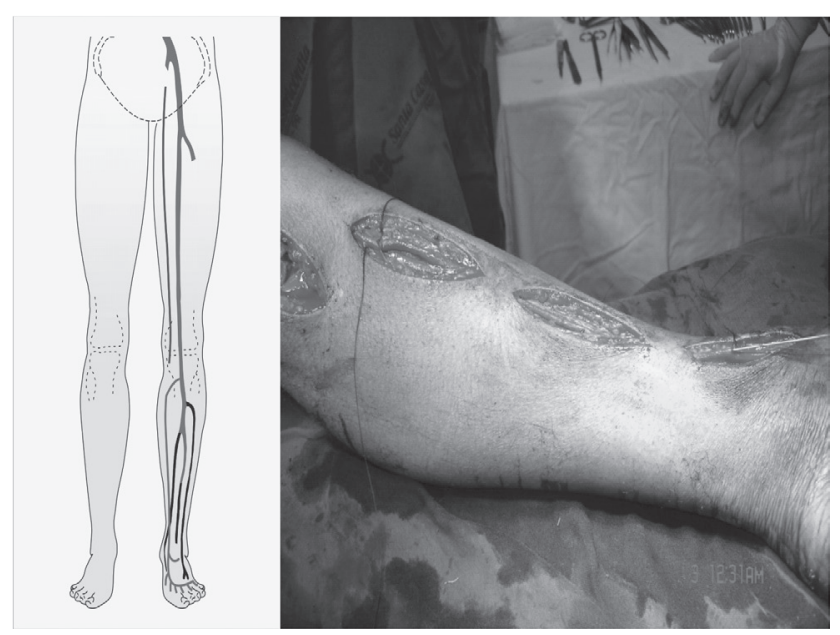

Figura 2 - Fluxo arterial derivado para o sistema venoso progride através da veia, cujas válvulas são destruídas pelo valvulótomo introduzido através de colaterais, até a altura do maléolo medial

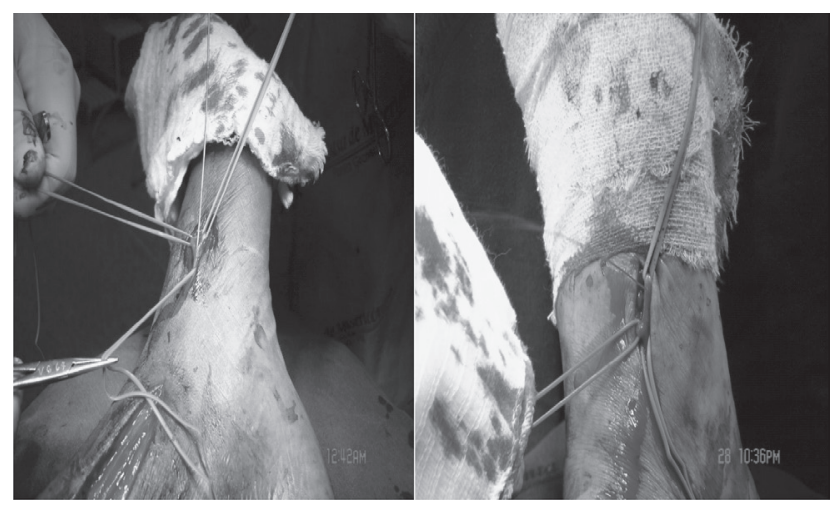

Figura 3 - Flebotomia no arco venoso dorsal, ao nível do primeiro espaço interdigital, completando a valvulotomia, permitindo o afluxo de sangue arterial ao dorso do pé 
Todas as colaterais da veia safena magna foram ligadas desde a anastomose arterial até a perfurante anterior do maléolo (Figura 4).

Dezoito pacientes com isquemia crítica sem leito arterial distal, dos quais $11 \mathrm{com} \mathrm{AO,} 6$ com TO e 1 com evolução tardia de aneurisma de artéria poplítea com trombose distal foram submetidos ao método. Dos 11 pacientes, 6 com $\mathrm{AO}$ apresentavam diabetes mellitus e, destes, 2 tinham insuficiência renal dependente de hemodiálise.

\section{Resultados}

Dos 18 pacientes "arterializados", 10 mantiveram suas extremidades $(55,6 \%)$. Seis cicatrizaram amputações menores, duas transmetatarsianas, duas de dedos e duas de falanges. Sete sofreram amputações maiores (38,9\%): três em nível de coxa e quatro em nível de perna. Houve um óbito (5,5\%) em paciente portador de diabetes mellitus e insuficiência renal crônica que desenvolveu septicemia por infecção ascendente.

Dos 11 pacientes com AO, 5 mantiveram a extremidade, 5 sofreram amputações maiores e 1 foi a óbito.

Dos seis pacientes com TO, cinco mantiveram a extremidade e um sofreu amputação maior.

O paciente com isquemia crítica causada pela trombose do aneurisma de artéria poplítea e obstrução do leito distal evoluiu com amputação em nível de coxa.

O seguimento médio dos pacientes cujos membros foram salvos foi de 695,6 dias (213 a 1.006). Dos pacientes com $\mathrm{AO}$, dois foram à óbito por comorbidades com enxerto pérvio. Dois, apesar de fecharem suas fístulas, mantiveram o membro, e um terceiro apresentava ainda sua fístula pérvia. Dos pacientes com TO, quatro tinham fístulas patentes, e um deles tinha uma fechada.

\section{Discussão}

Os bons resultados da cirurgia estão relacionados à indicação precisa, estudo pré-operatório arterial e venoso da extremidade em risco e detalhes de técnica operatória. É imprescindível a presença de pulso e frêmito ao nível do arco venoso dorsal, manutenção das veias do pé a partir da perfurante anterior do maléolo e integridade do sistema venoso profundo do membro que serve como "rota de fuga" ao hiperfluxo gerado pela fístula (Figura 5).

Root e Cruz ${ }^{21}$ e Matolo ${ }^{22}$ demonstraram experimentalmente que as fístulas término-laterais permitiam um bom retorno venoso e melhores resultados, que as términoterminais, que por sobrecarga venosa, levavam a edema, à equimose e à necrose.

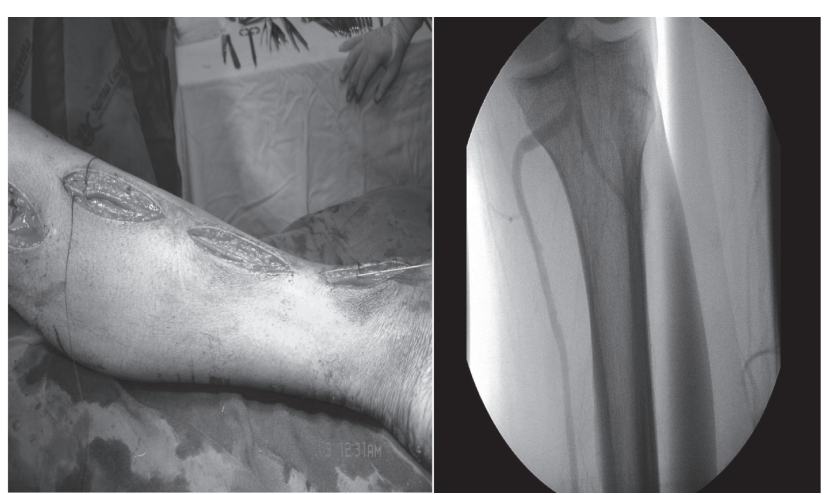

Figura 4 - Ligadura das colaterais da veia safena magna, desde a anastomose arterial, até a perfurante anterior do maléolo, demonstrada também na arteriografia

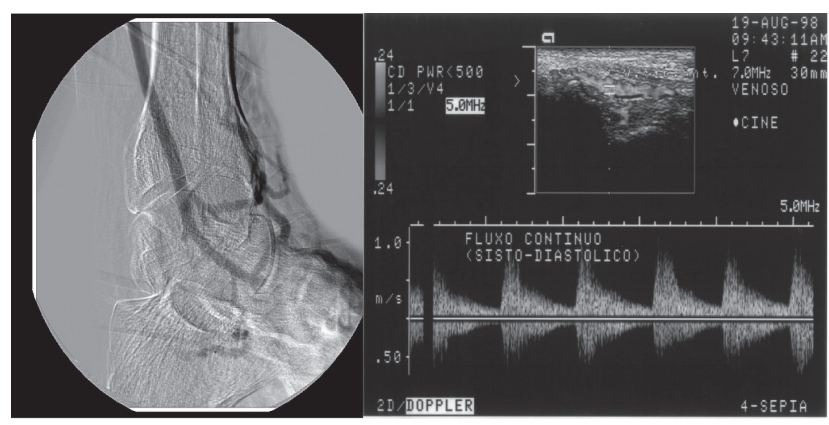

Figura 5 - Arteriografia mostrando difusão do contraste para o sistema profundo e duplex com fluxo sisto-diástólico, ao nível do arco venoso dorsal do pé

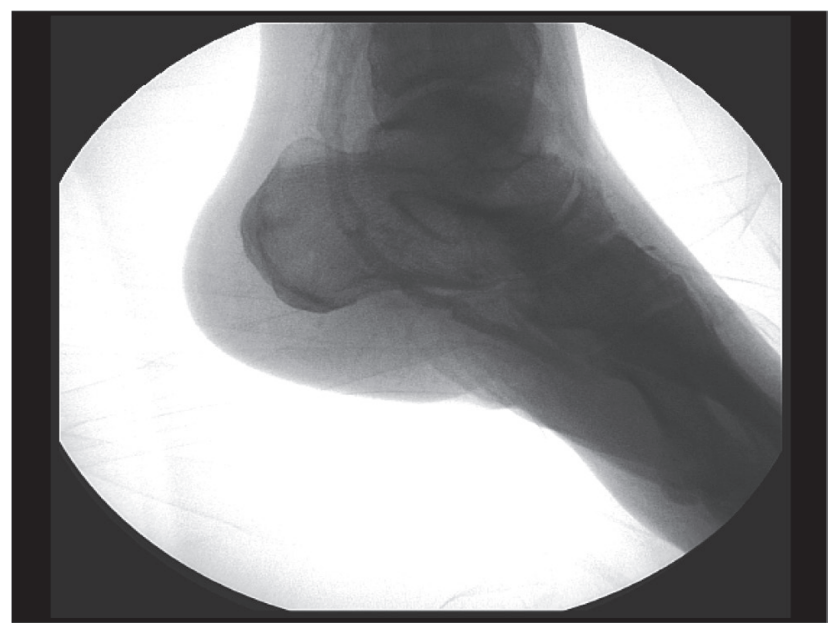

Figura 6 - Arteriografia pós-operatória com enchimento do arco plantar do pé e da veia safena parva

A avaliação pré-operatória pelo mapeamento dúplex, além de escolher a melhor artéria doadora, deve mostrar sistema venoso profundo, safena magna e arco venoso do pé íntegros. 
A perfurante anterior do maléolo deriva parte do fluxo para as veias tibiais anteriores, e parte para as veias do dorso proximal do pé (Figura 5).

Lofgren et al..$^{23}$ demonstraram que a injeção de látex azul no arco venoso dorsal, entre o primeiro e segundo metatarsiano, escoava para as veias profundas e superficiais da porção proximal. Notaram ainda que mais da metade das perfurantes venosas (entre 6 e 12), que comunicam os sistemas venosos superficial e profundo, são desprovidas de válvulas, permitindo o fluxo em ambas as direções. A perfurante de maior importância é a do primeiro espaço interdigital, com aproximadamente $3 \mathrm{~mm}^{24}$. Observou-se em arteriografias pós-operatórias, além do descrito, o enchimento do arco plantar do pé e da veia safena parva (Figura 6).

Embora a TO acometa tanto as veias quanto as artérias, raramente as safenas magna e parva estão acometidas pelo processo inflamatório ${ }^{25}$.

A utilização da safena magna in situ permite "arterializar" o arco venoso do pé com apenas uma anastomose, sem a retirada da veia de seu leito original, dispensando a confecção de túnel. No entanto, os resultados dependem mais do tipo de pacientes tratado do que propriamente da técnica utilizada.

Em 2006, resultados semelhantes aos deste trabalho foram encontrados em levantamento de 56 publicações disponíveis na literatura mundial, que utilizaram o procedimento para tratamento de isquemia crítica sem leito distal, por diferentes técnicas. Sete trabalhos submetidos à metaanálise perfizeram um total de 228 pacientes com 231 extremidades tratadas e um percentual de salvamento de $71 \%$, com cicatrização de lesões, pequenas amputações e melhora da dor em repouso: 140 casos com $\mathrm{AO}$ e 91 com $\mathrm{TO}^{26}$.

Concluiu-se que a revascularização distal do membro com isquemia crítica por meio da inversão do fluxo venoso do pé, pela "arterialização" da safena in situ, deve ser considerada para salvamento de extremidade com isquemia crítica sem leito arterial distal.

\section{Referências}

1. Lengua F, Herrera EZ, Kunlin J. Nuevos documentos experimentales de inversion circulatoria em miembro isquemico y de inyeccion retrograda em piezas anatomicas. Diagnostico. 1984;13:77-86.

2. Lengua F, Helfner L. Técnica de arterialization de I ares venosa del pie. Rev Sand Polic. 1984;35:203-10

3. Lengua F, Nuss JM, Lechner R, Kunlin J. Arterialization of the venous network of the foot through a bypass in severe arteriopathic ischemia. J Cardiovasc Surg. 1984; 25:357-60

4. Lengua F, Nuss JM, Buffet JM, Lechner R. Etude comparative de deux modalités d'arterialisation des veines du pied en ischémie critique. J Chir. 1993;130:12-9.
5. Lengua F. Le pontage d'artérialisation veineuse distale peutil être bénéfique au pied diabétique avec nécrose? Chirurgie. 1994-1995;120:143-52.

6. Lengua F, Cohen R, Huillier BL, Buffet JM. Arteriovenous revascularization for lower limb salvage in unreconstructible arterial occlusive disease (long term outcome). Vasa. 1995;24:261-9.

7. Lengua F, Madrid A La, Acosta C, et al. L'arterialisation des veines du pied pour sauvetage de membre chez l'artéritique. Technique et resultats. Ann Chir. 2001;126:629-38.

8. Pokrovski AV, Dan VN, Khorovets AG, Chupin AV. Arterialization of venous blood flow in the foot in the treatment of severe ischaemia in patients with crural arterial occlusions and non-functioning plantar arch. Khirurgiia. 1990;5:35-42.

9. Pokrovski AV, Dan VN, Khorovets AG, Chupin AV. Arterialization of the foot venous system in the treatment of the critical lower limb ischaemia and distal arterial bed occlusion. An Vasc Surg. 1996; $4: 73-93$

10. Chen XS, Lin T, Chen DL, Guan YB. Venous arterialization in the treatment of extensive arterial occlusion of lower extremities. J Surg Concepts Pract. 1998;3:219-21.

11. Taylor RS, Belli AM, Jacob S. Salvage of critically ischaemic limbs. Lancet. 1999;354:1962-5.

12. Engelke C, Morgan RA, Quarmby JW, Taylor RS, Belli AM. Distal venous arterialization for lower limb salvage: angiographic appearances and interventional procedures. Radiographics. 2001;21:1239-50.

13. Rowe VL, Hood DB, Liphan J, et al. Initial experience with dorsa venous arch arterialization for limb salvage. Ann of Vasc Surg. 2002; 16:187-92.

14. Ozbeck C, Kestelli M, Emrecan B, et al. A novel approach: ascending venous arterialization for atherosclerosis obliterans. Eur I Vasc Endovas Surg. 2005;29:47-51.

15. Gavrilenko AV, Skrylev SI. Long-term results of venous blood flow arterialization of the leg and foot in patients with critical lower limb ischemia. Angiol Sosud Khir. 2007;13:95-103.

16. Keshelawa G, Gigilashvili K, Chkholaria A, Pagava G, Janashia G, Beselia K. Foot venous system arterialization for salvage of nonreconstructable acute ischemic limb: a case report. J Vasc Nurs. 2009;27:13-6.

17. Busato $C R$, Utrabo CAL, Housome JK, Gomes RZ. Arterialização do arco venoso do pé para tratamento da isquemia crítica sem leito distal. Cir Vasc \& Angiol. 1999;15:117-21.

18. Busato CR, Utrabo CAL, Gomes RZ, et al. Arterialização do arco venoso do pé para tratamento da tromboangeíte obliterante. J Vasc Bras. 2008;7:267-71

19. Gasparis AP, Noor S, Da Silva MS, Tassiopoulos AK, Semel L. Distal venous arterialization for limb salvage: a case report. Vasc Endovasc Surg. 2002;36:469-72.

20. Lozano A, Melon J, Ruiz-Grande F, et al. Arterialización venosa distal en cirugía de salvación de extremidad. Resultados preliminaries Angiologia. 2002;54:204-26.

21. Root HD, Cruz AB. Effects of an arteriovenous fistula on the devascularized limb. JAMA. 1965;191:645-8.

22. Matolo NM, Cohen SE, Wolfmann EF Jr. Use of an arteriovenous fistula for treatment of the severely ischemic extremity: experimental evaluation. Ann Surg. 1976;5:622-5. 
23. Lofgren EP, Myers TT, Lofgren KA, Kuster G. The venous valves of the foot and ankle. Surg Gynecol Obstet. 1968;8:289-90.

24. Garrido MBM. Anatomia médico-cirúrgica do sistema venoso dos membros inferiores. In: Maffei FHA. Doenças vasculares periféricas. 3. ed. Rio de Janeiro: Medsi; 2002. v. 1. p. 133-67.

25. Kauffman P. Tromboangeíte obliterante. In: Maffei FHA. Doenças vasculares periféricas. 3. ed. Rio de Janeiro: Medsi; 2002. v. 2. p. $1271-9$.

26. Lu XW, Idu MM, Ubbink DT, Legemate DA. Meta-analysis of the clinical effectiveness of venous arterialization for salvage of critically ischaemic limbs. Eur J Vasc Endovasc Surg. 2006;31:493-9.
Correspondência: César Roberto Busato Rua Saldanha da Gama, 425 CEP 84015-130 - Ponta Grossa (PR), Brasil E-mail: crbusato@brturbo.com.br

Contribuições dos autores Concepção e desenho do estudo: CRB, CALU e CDB Análise e interpretação dos dados: CRB Coleta de dados: CRB, CALU, RZG, JKH e EH Redação do artigo: CRB, DMMC e CDB Revisão crítica do texto: $C R B$ Aprovação final do artigo: CRB, CALU, RZG, JKH, EH e DMMC Análise estatística: não houve Responsabilidade geral pelo estudo: CRB Informações sobre financiamento: não houve Todos os autores leram e aprovaram a versão final submetida ao J Vasc Bras. 